\title{
Size effects in the particle-reinforced metal-matrix composites
}

\author{
S. H. Chen and T. C. Wang, Beijing, China \\ (Received August 20, 2001; revised September 10, 2001)
}

\begin{abstract}
Summary. Many experimental observations have shown the influences of particle size on the mechanical properties of the particle-reinforced metal-matrix composite. However, the conventional theory cannot explain the phenomena because no length scale parameters are included in the conventional theory. In the present paper, the strain gradient theory proposed by Chen and Wang [32] is used, and a systematic research of the particle size effect in the particle-reinforced metal-matrix composite is carried out. Many composite factors, such as the particle size, the particle aspect ratio, the Young's modulus ratio of the particle to the matrix material, particle volume fraction and the strain hardening exponent of the matrix material, are investigated in detail. Two kinds of particle shapes, spheroidal particle and cylindrical particle, are considered to check the strength dependence of the particle shapes. Calculation to the special materials used by Ling [9] has been done, and the calculation results are consistent with the experimental results in Ling [9]. The material length scale parameter is predicted.
\end{abstract}

\section{Introduction}

Particle-reinforced metal matrix composites (PMMC) are promising candidates for a number of aerospace and automotive applications due to their higher specific stiffness, specific strength and better wear resistance. It is well known that the mechanical behavior of this class of materials is significantly affected by their microstructure, such as the Young's modulus of the particle, the particle aspect ratio, the particle volume fraction and size effect, as well as the strain hardening exponent of the matrix material. During the past two decades, many attempts have been made to explore the relationship between microstructure and deformation behavior in PMMC. Continuum models including the cell models [1], [2] gave some detailed quantitative information about the composite strength. The composite always shows strengthening because a higher triaxiality stress exists within the matrix region near the particle surface under loading [1]. The particulate aspect ratio and the volume fracture, as well as the strain hardening exponent of the matrix material had important influences on the composite properties, and some quantitative relations were developed by using cell models. The predicted results are consistent with experimental results. In addition, a self-consistent analytical model has been used successfully to predict the behavior of the PMMC [3]. More recently, a systematic experimental research for the metallic fiber reinforced $\mathrm{Al}$-alloy matrix for a series of the volume fraction of fiber was carried out [4]. On the researches of the particle size effects, the experimental results [5]-[9] showed that the strength of the PMMC was sensitive to the particle size. The conclusion was that the smaller the particle, the higher the composite strength. In order to predict the particle size effects, some analytical models were developed [10], [11]. It is well known that the conventional elastic-plastic theory cannot be used to pre- 
dict the particle size effect effectively, because no length scale parameter is included in the theory. Besides PMMC, these size effects have been found in many research regions, such as in the micro-indentation test [12], in the micro-torsion test of thin copper wire [13], in the ultrathin beam bending test [14] as well as the interface separation of metal/ceramic system [15].

In order to describe the size effects, several strain gradient theories have been developed [16]-[18]. Fleck and Hutchinson [16] developed a phenomenological strain gradient theory based on the reduced couple stress theory, and a material length scale was introduced for dimensional grounds. In order to explain experimental findings of indentation and fracture [12], [15], Fleck and Hutchinson [17] gave another strain gradient theory, in which two kinds of length parameters were introduced. Wei [19], [20] has used the strain gradient theory in [17] to investigate crack tip field and size effects in the PMMC. A rate-dependent strain gradient crystal plasticity formulation was developed by Shu and Fleck [21] and was used to investigate the microscopic details of the deformation of a whisker-reinforced metal-matrix composite [22]. Bassani et al. [23] used both discrete dislocation plasticity and a nonlocal continuum crystal plasticity theory to investigate a two-dimensional model composite with elastic reinforcements in a crystalline matrix, which is subjected to macroscopic shear. Nix and Gao [24] started from the Taylor relation and gave one kind of hardening law for gradient plasticity. Motivated by the hardening law, Gao et al. [18] proposed a mechanism-based theory of strain gradient plasticity (MSG).

However, all the above strain gradient plasticity theories introduce the higher order stress which is required for this class of strain gradient theories to satisfy the Clausius-Duhem thermodynamic restrictions on the constitutive model for second deformation gradients [25]. In comparison, no work conjugate of strain gradient has been defined in the alternative gradient theories [26]-[29]. Retaining the essential structure of conventional plasticity and obeying thermodynamic restrictions, Acharya and Bassani [30] concluded that the only possible formulation is a flow theory with strain gradient effects represented as an internal variable, which acts to increase the current tangent-hardening modulus. Chen and Wang [31] established a concrete hardening law based on the incremental version of conventional $J_{2}$ deformation theory, which allows the problem of incremental equilibrium equations to be stated without higher-order stress, higher-order strain rate or extra boundary conditions. The new hardening law has been used to investigate microtwisting and microbending experiments. The predictions based on the hardening law agree well with experimental data. Subsequently, Chen and Wang [32] have proposed a new strain gradient theory with independent microrotation degrees of freedom, $\omega_{i}$, which has no relation to the displacement $u_{i}$. While only the rotation gradient is considered, the phenomena found in the thin-wire torsion and ultra-thin beam bending tests can be explained successfully. And the theory only considering the rotation gradient was used to analyse the crack tip asymptotic field [33], [34]. It is found that while the fracture and indentation problems are considered, the new hardening law proposed by Chen and Wang [31] must be used, in which the stretch gradient is introduced. Using the new strain gradient theory and hardening law, fracture and indentation problems have been successfully investigated [35], [36], and the results explain the phenomena found in the experiments [15], [12] very well.

In the present paper, the hardening law in [31] and the strain gradient theory in [32] are used to simulate the mechanical response of the PMMC. A detailed analysis of the particle size effect is carried out. Adopting the strain gradient plasticity theory, the composite stressstrain curves will depend on the following parameters: the Young's modulus of both particle and matrix; particle volume fraction; particle aspect ratio; strain hardening exponent of matrix material; a normalized particle volume with the material length scale parameter. A cell 
model is adopted and two kinds of particle shapes: spheroid and cylinder are considered. Finally, using analysis results to the experiments, the material length scale parameter is predicted.

\section{Strain gradient theory}

\subsection{Review of the general couple stress theory}

In the general couple stress theory, the micro-rotation vector, $\omega$, is treated as an independent kinematic quantity with no direct dependence upon $\boldsymbol{u}$ and distinct from the material rotation $\boldsymbol{\theta} \equiv(1 / 2) \operatorname{curl} \boldsymbol{u} . \boldsymbol{\sigma}$ denotes the symmetric part of the Cauchy stress and $\boldsymbol{\tau}$ denotes the antisymmetric part of the Cauchy stress, $\boldsymbol{m}$ denotes the overall couple stress tensor.

Neglecting the body forces and body couples, the principle of virtual work for the general couple stress theory is

$\left.\int_{V}\left(\sigma_{i j}+\tau_{i j}\right) \delta \gamma_{i j}+m_{i j} \delta \chi_{i j}\right] \mathrm{d} V=\int_{S}\left[T_{i} \delta u_{i}+q_{i} \delta \omega_{i}\right] \mathrm{d} S$,

where $\chi_{i j}=\omega_{i, j}$ is the work-conjugated curvature tensor and $\gamma_{i j}=u_{i, j}+e_{i j k} \omega_{k}$ is called the relative displacement gradient tensor. $V$ is the volume of the body and $S$ is the surface of it.

The above virtual work statement can be rearranged to the form

$\int_{V}\left(\sigma_{i j} \delta \varepsilon_{i j}+\tau_{i j} \delta \alpha_{i j}+m_{i j} \delta \chi_{i j}\right] \mathrm{d} V=\int_{S}\left[T_{i} \delta u_{i}+q_{i} \delta \omega_{i}\right] \mathrm{d} S$

where the symmetric tensor $\varepsilon_{i j}$ is the usual strain tensor,

$\varepsilon_{i j}=\frac{u_{i, j}+u_{j, i}}{2}$

and the anti-symmetric tensor $\alpha_{i j}$ is the relative rotation tensor

$\alpha_{i j}=e_{i j k} \omega_{k}-\left(u_{j, i}-u_{i, j}\right) / 2=e_{i j k}\left(\omega_{k}-\theta_{k}\right)$.

Via the divergence theorem, the left side in Eq. (2) can be written as

$$
\begin{aligned}
\int_{V}\left(\sigma_{i j} \delta \varepsilon_{i j}+\tau_{i j} \delta \alpha_{i j}+m_{i j} \delta \chi_{i j}\right] \mathrm{d} V= & \int_{S}\left(\sigma_{i j}+\tau_{i j}\right) n_{j} \delta u_{i} \mathrm{~d} S-\int_{V}\left(\sigma_{i j}+\tau_{i j}\right)_{, j} \delta u_{i} \mathrm{~d} V \\
& +\int_{S} m_{i j} n_{j} \delta \omega_{i} \mathrm{~d} S+\int_{V} \tau_{j k} e_{i j k} \delta \omega_{i} \mathrm{~d} V-\int_{V} m_{i j, j} \delta \omega_{i} \mathrm{~d} V,
\end{aligned}
$$

then, the equilibrium relation of force in $V$ is

$t_{i j, j}=\sigma_{i j, j}+\tau_{i j, j}=0$,

and moment equilibrium is

$\tau_{j k}=\frac{1}{2} e_{i j k} m_{i p, p}$.

Here, a comma indicates a partial derivative with respect to a Cartesian coordinate and a repeated suffix denotes summation over 1 to 3 . A subscript index can take the value of 1,2 or 3 , and $e_{i j k}$ denotes the usual permutation symbol.

Traction equilibrium on the surface $S$ of the body is

$$
T_{i}=\left(\sigma_{i j}+\tau_{i j}\right) n_{j}, \quad q_{i}=m_{i j} n_{j} .
$$


The strain energy density function $w$ in the general couple stress theory depends upon the strain tensor $\boldsymbol{\varepsilon}$, the curvature tensor $\boldsymbol{\chi}$ and the relative rotation $\boldsymbol{\alpha}$ as follows:

$w(\boldsymbol{\varepsilon}, \boldsymbol{\chi}, \boldsymbol{\alpha})=\int_{0}^{\varepsilon_{i j}} \sigma_{i j} \mathrm{~d} \varepsilon_{i j}+\int_{0}^{\chi_{i j}} m_{i j} \mathrm{~d} \chi_{i j}+\int_{0}^{\alpha_{i j}} \tau_{i j} \mathrm{~d} \alpha_{i j}$

then, the constitutive relations are

$\sigma_{i j}=\frac{\partial w}{\partial \varepsilon_{i j}}, \quad m_{i j}=\frac{\partial w}{\partial \chi_{i j}}, \quad \tau_{i j}=\frac{\partial w}{\partial \alpha_{i j}}$.

\subsection{Strain gradient theory}

The strain gradient theory given by Chen and Wang [32] is based on the framework of general couple stress theory. It is different from the theory proposed by Fleck et al. [16], which is based on a reduced couple stress theory. The micro-rotation vector $\boldsymbol{\omega}$, which is the sum of the material rotation vector $\boldsymbol{\theta}$ plus the particle relative rotation vector with respect to the material, is an independent quantity with no direct dependence upon $\boldsymbol{u}$, i.e. $\boldsymbol{\omega} \neq \boldsymbol{\theta}$. So that the relative rotation tensor $\boldsymbol{\alpha}$ does not equal zero, which is different from the other existing theories.

We postulate that the strain energy density $w$ depends only upon the strain tensor $\varepsilon$ and the curvature tensor $\boldsymbol{\chi}$, i.e. the relative rotation tensor $\boldsymbol{\alpha}$ has no contributions to the strain energy density $w$. It follows

$\tau_{i j}=\frac{\partial w}{\partial \alpha_{i j}}=0$.

Since the anti-symmetric part of Cauchy stress vanishes in the present theory, in the following sections, the symmetric part of Cauchy stress is called Cauchy stress directly.

The deviatoric part $s_{i j}$ of Cauchy stress and deviatoric part $m_{i j}^{\prime}$ of couple stress are defined as the work conjugates of $\varepsilon_{i j}^{\prime}, \chi_{i j}^{\prime}$, respectively; $\sigma_{m}$ and $m_{m}$ are defined as the work conjugates of $\varepsilon_{m}$ and $\chi_{m}$, respectively, giving

$\delta w=s_{i j} \delta \varepsilon_{i j}^{\prime}+m_{i j}^{\prime} \delta \chi_{i j}^{\prime}+\sigma_{m} \delta \varepsilon_{m}+m_{m} \delta \chi_{m}$,

where $s_{i j} \equiv \sigma_{i j}-(1 / 3) \delta_{i j} \sigma_{k k}$ and $m_{i j}^{\prime} \equiv m_{i j}-(1 / 3) \delta_{i j} m_{k k}$.

Equation (12) enables one to determine $s_{i j}, m_{i j}^{\prime}, \sigma_{m}$ and $m_{m}$ in terms of the strain and curvature states of the solid as

$s_{i j}=\frac{\partial w}{\partial \varepsilon_{i j}^{\prime}} \quad m_{i j}^{\prime}=\frac{\partial w}{\partial \chi_{i j}^{\prime}} \quad \sigma_{m}=\frac{\partial w}{\partial \varepsilon_{m}} \quad m_{m}=\frac{\partial w}{\partial \chi_{m}}$.

According to the work by Fleck et al. [13], [16], it is mathematically convenient to assume that the strain energy density $w$ depends only upon the single scalar strain measure $E_{e}$, where

$E_{e}^{2}=\varepsilon_{e}^{2}+l_{c s}^{2} \chi_{e}^{2}$.

$E_{e}$ is called the generalized effective strain in the present paper. $l_{c s}$ is an intrinsic material length, which reflects the size effects of the rotation gradient on the material behaviors.

$\Sigma_{e}$ is the work conjugate of $E_{e}$ and defined by

$\Sigma_{e}=\frac{\mathrm{d} w\left(E_{e}\right)}{\mathrm{d} E_{e}}$. 
Then, Eqs. (13) can be written as

$s_{i j}=\frac{2 \Sigma_{e}}{3 E_{e}} \varepsilon_{i j}^{\prime}, \quad m_{i j}^{\prime}=\frac{2 \Sigma_{e}}{3 E_{e}} l_{c s}^{2} \chi_{i j}^{\prime}, \quad \sigma_{m}=K \varepsilon_{m}, \quad m_{m}=K_{1} l_{c s}^{2} \chi_{m}$,

where $K$ is the volumetric modulus; $K_{1}$ is the bend-torsion volumetric modulus and

$\Sigma_{e}=\sqrt{\sigma_{e}^{2}+l_{c s}^{-2} m_{e}^{2}}$,

$\sigma_{e}^{2}=\frac{3}{2} s_{i j} s_{i j}, \quad m_{e}^{2}=\frac{3}{2} m_{i j}^{\prime} m_{i j}^{\prime}$.

The equilibrium relations of Eqs. (6) and (7) can be written as

$\sigma_{i j, j}=0$,

$m_{i j, j}=0$.

The traction boundary conditions for force and moment are

$\sigma_{i j} n_{j}=T_{i}^{0} \quad$ on $\quad S_{T}$,

$m_{i j} n_{j}=q_{i}^{0} \quad$ on $\quad S_{q}$.

The additional boundary conditions are

$u_{i}=u_{i}^{0} \quad$ on $\quad S_{u}$,

$\omega_{i}=\omega_{i}^{0} \quad$ on $\quad S_{\omega}$.

\subsection{New hardening law}

The hardening relationship in conventional plasticity theory can be expressed as follows:

$\sigma_{e}=A\left(\varepsilon_{e}\right)=\frac{\mathrm{d} w\left(\varepsilon_{e}\right)}{\mathrm{d} \varepsilon_{e}}$,

and the incremental form of Eq. (25) is

$\dot{\sigma}_{e}=A^{\prime}\left(\varepsilon_{e}\right) \dot{\varepsilon}_{e}$,

where $A^{\prime}\left(\varepsilon_{e}\right)$ is the tangent hardening modulus in the incremental version of conventional $J_{2}$ deformation theory.

While only the rotation gradient is considered, the relation between $\Sigma_{e}$ and $E_{e}$ is taken as

$\Sigma_{e}=A\left(E_{e}\right), \quad \Sigma_{e} \geq \sigma_{Y}$

$\Sigma_{e}=3 \mu E_{e}, \quad \Sigma_{e}<\sigma_{Y}$,

where $\sigma_{Y}$ is the yield stress and $\mu$ is the shear modulus.

While the stretch gradient is considered, the hardening strength is related with not only the density of statistically stored dislocation but also the density of geometrically necessary dislocation. Instructed by this idea, the new incremental hardening relationship similar to that in [31] instead of Eq. (27) is proposed:

$$
\begin{array}{ll}
\dot{\Sigma}_{e}=A^{\prime}\left(E_{e}\right)\left(1+\frac{l_{1} \eta_{1}}{E_{e}}\right)^{1 / 2} \dot{E}_{e}=B\left(E_{e}, l_{1} \eta_{1}\right) \dot{E}_{e} & \Sigma_{e} \geq \sigma_{Y}, \\
\dot{\Sigma}_{e}=3 \mu \dot{E}_{e} & \Sigma_{e}<\sigma_{Y}
\end{array}
$$


where $B\left(E_{e}, l_{1} \eta_{1}\right)$ is the hardening function including the effect of the stretch gradient; $l_{1}$ is the second intrinsic material length associated with the stretch gradient. $\eta_{1}$ is the effective stretch gradient defined as follows [37]:

$\eta_{i j k}=u_{k, i j}$

$\eta_{i j k}^{\prime}=\eta_{i j k}-\frac{1}{4}\left(\delta_{i k} \eta_{j p p}+\delta_{j k} \eta_{i p p}\right)$

$\eta_{i j k}^{s}=\frac{1}{3}\left(\eta_{i j k}^{\prime}+\eta_{j k i}^{\prime}+\eta_{k i j}^{\prime}\right)$,

$\eta_{i j k}^{(1)}=\eta_{i j k}^{s}-\frac{1}{5}\left(\delta_{i j} \eta_{k p p}^{s}+\delta_{j k} \eta_{i p p}^{s}+\delta_{k i} \eta_{j p p}^{s}\right)$

$\eta_{1}=\sqrt{\eta_{i j k}^{(1)} \eta_{i j k}^{(1)}}$

It must be noted that on each incremental step both the effective strain $\varepsilon_{e}$ and the effective stretch gradient $\eta_{1}$ can be obtained from the updated displacement fields $u_{i}$; the effective rotation gradient $\chi_{e}$ can be obtained from the updated rotation fields $\omega_{i}$. Hence, $\eta_{1}$ is only a given parameter in Eq. (28), and it doesn't invoke higher-order stress or higher-order strain rates.

\subsection{Incremental constitutive relations}

While the stretch gradient is introduced through the hardening law, Eq. (28), which is in the incremental form, the constitutive relations can be written in the incremental form as follows:

$$
\begin{array}{ll}
\dot{\sigma}_{i j}=2 \mu \dot{\varepsilon}_{i j}^{\prime}+K \dot{\varepsilon}_{m} \delta_{i j} & \Sigma_{e}<\sigma_{Y} \\
\dot{m}_{i j}=2 \mu l_{c s}^{2} \dot{\chi}_{i j}^{\prime}+K_{1} l_{c s}^{2} \dot{\chi}_{m} \delta_{i j} & \\
\dot{\sigma}_{i j}=\frac{2 \Sigma_{e}}{3 E_{e}} \dot{\varepsilon}_{i j}^{\prime}+\frac{2 \dot{\Sigma}_{e}}{3 E_{e}} \varepsilon_{i j}^{\prime}-\frac{2 \Sigma_{e}}{3 E_{e}{ }^{2}} \varepsilon_{i j}^{\prime} \dot{E}_{e}+K \dot{\varepsilon}_{m} \delta_{i j} & \Sigma_{e} \geq \sigma_{Y} . \\
\dot{m}_{i j}=\frac{2 \Sigma_{e}}{3 E_{e}} l_{c s}^{2} \dot{\chi}_{i j}^{\prime}+\frac{2 \dot{\Sigma}_{e}}{3 E_{e}} l_{c s}^{2} \dot{\chi}_{i j}^{\prime}-\frac{2 \Sigma_{e}}{3 E_{e}{ }^{2}} l_{c s}^{2} \chi_{i j}^{\prime} \dot{E}_{e}+K_{1} l_{c s}^{2} \dot{\chi}_{m} \delta_{i j} &
\end{array}
$$

Here, $\Sigma_{e}$ is the integration of $\dot{\Sigma}_{e}$, and $\dot{\Sigma}_{e}$ is expressed by Eq. (28).

\section{Finite element formulation and calculation model}

In this section, the finite element formulations are presented for the strain gradient theory used in the present paper. The principle of virtual work requires

$\int_{V}\left(\sigma_{i j} \delta \varepsilon_{i j}+m_{i j} \delta \chi_{i j}\right) \mathrm{d} V=\int_{S}\left(t_{k} \delta u_{k}+q_{k} \delta \omega_{k}\right) \mathrm{d} S$.

The virtual strains $\delta \varepsilon_{i j}$ are related to the virtual displacements $\delta u_{k}$, and $\delta \chi_{i j}$ are related to the virtual rotation vector $\delta \omega_{k} . t_{k}$ is the surface stress traction and $q_{k}$ is the surface torque traction.

The nodal displacements and rotation vectors have to be solved incrementally due to the incremental hardening law, i.e. Eq. (28). Therefore, the nodal displacements and the rotation 
vectors are solved for each loading step by rewriting the principle of virtual work, Eq. (36), about the current solution as

$$
\begin{aligned}
& \int_{V}\left(\Delta s_{i j} \delta \varepsilon_{i j}^{\prime}+\Delta \sigma_{m} \delta \varepsilon_{k k}+\Delta m_{i j}^{\prime} \delta \chi_{i j}^{\prime}+\Delta m_{m} \delta \chi_{k k}\right) \mathrm{d} V-\int_{S}\left(\Delta t_{k} \delta u_{k}+\Delta q_{k} \delta \omega_{k}\right) \mathrm{d} S \\
& \left.=-\int_{V} s_{i j} \delta \varepsilon_{i j}^{\prime}+\sigma_{m} \delta \varepsilon_{k k}+m_{i j}^{\prime} \delta \chi_{i j}^{\prime}+m_{m} \delta \chi_{k k}\right) \mathrm{d} V+\int_{S}\left(t_{k} \delta u_{k}+q_{k} \delta \omega_{k}\right) \mathrm{d} S
\end{aligned}
$$

where the superscript prime denotes the deviatoric quantities, $\Delta$ on the left-hand side stands for increments, whereas the right-hand side involves the current quantities.

\subsection{Choice of elements}

Many researchers [19], [20], [38], [39] have found that the choice of the element for gradient plasticity is complicated and in particular quite sensitive to details of the constitutive relation. Xia and Hutchinson [39] have discussed some choices of finite elements for strain gradient plasticity with the emphasis on plane strain cracks. Several elements have been developed for the phenomenological strain gradient plasticity theory to investigate the crack tip field, microindentation experiments and stress concentrations around a hole. A review of these elements can be found in [40].

For the two-dimensional case, such as the problem of plane strain and the axis-symmetry, second-order element can be used, such as the eight-node and nine-node elements. In the present paper, nine-node elements have been used to analyze the PMMC problem. The displacement and rotation vectors in the element are interpolated through the shape function, whereas the strain and the rotation gradient tensors in the element are then obtained from the displacement derivatives and the micro-rotation vector's derivatives. This element is only suitable for solids with vanishing higher-order stress traction on the surface. For example, the element has worked very well in the fracture analysis of strain gradient plasticity [19], where the higherorder stress tractions vanish on the crack face and on the remote boundary. This element also works well in the study of microindentation experiments [36] because the higher-order stress tractions are zero on the indented surface. Since the strain gradient theory used in the present paper does not include higher-order stress and higher-order stress tractions, these kinds of elements will work well in the present study.

\subsection{The nodal degrees of freedom}

It is convenient to express the field quantities in terms of the circular cylindrical coordinate system $(r, \theta, z)$ as shown in Fig. 1. Both the geometry and loading are axis-symmetric, and without loss of generality we consider the section of $\theta=0$.

Since the independent parameter $\omega_{i}$ is introduced in addition to the displacement $u_{i}$ in the present strain gradient theory, which is different from the theory proposed by Fleck and Hutchinson [16], one node has three degrees of freedom, i.e. $u_{i r}, u_{i z}$ and $\omega_{i \theta}$ for the axis-symmetric indentation case. The displacement field and the rotation vector field can be obtained through the shape function $N_{i}$, the nodal displacements and nodal rotation vectors, i.e.,

$$
\begin{aligned}
& u_{r}=\sum_{i=1}^{n} N_{i} u_{i r}, \\
& u_{z}=\sum_{i=1}^{n} N_{i} u_{i z}, \\
& \omega_{\theta}=\sum_{i=1}^{n} N_{i} \omega_{i \theta} .
\end{aligned}
$$




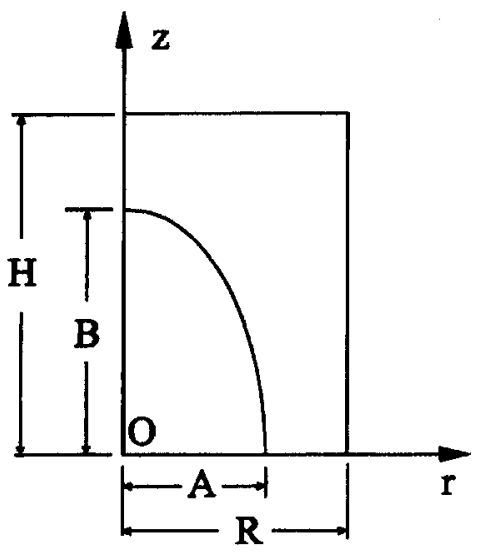

Fig. 1. Cell model and the coordinate system

\subsection{Calculation model}

Consider two kinds of particles, spheroid and cylinder. The simplified cell models are shown in Fig. 1. For the axial-symmetrical condition, only one fourth of the material region needs to be considered as shown in Fig. 1. The normalized cell sizes for the spheroidal particles are

$A=\left(\frac{3 \varkappa}{4 \pi}\right)^{1 / 3} \frac{V_{P}^{1 / 3}}{l_{1}}, \quad B=\frac{A}{\varkappa}, \quad R=\left(\frac{\varkappa}{2 \pi f_{P}}\right)^{1 / 3} \frac{V_{P}^{1 / 3}}{l_{1}}, \quad H=\frac{R}{\chi}$,

where $A, B, R$ and $H$ are shown in Fig. $1 ; V_{P}$ and $f_{P}$ are the particle volume and volume fraction, respectively, and

$\varkappa=\frac{A}{B}=\frac{R}{H}$

are the particle and cell aspect ratios. Note that the cell volume can not take unity as usual because the reference length is $l_{1}$, instead of the usual cell size. The relation between the two material length scales is taken as $l_{c s}=l_{1}=L$ in the present paper. Therefore, three independent parameters for the cell geometry description are needed, instead of only two independent parameters for the usual cell model description. From dimensional analysis, an additional composite parameter, $V_{P}^{1 / 3} / l_{1}$, describing the cell size and the strain gradient effects, must appear in the analysis inevitably.

For the cylindrical particle case, we have

$A=\left(\frac{\varkappa}{\pi}\right)^{1 / 3} \frac{V_{P}^{1 / 3}}{l_{1}}, \quad B=\frac{A}{\varkappa}, \quad R=\left(\frac{\varkappa}{\pi f_{P}}\right)^{1 / 3} \frac{V_{P}^{1 / 3}}{l_{1}}, \quad H=\frac{R}{\varkappa}$.

The boundary conditions as shown in Fig. 1 are described as

$$
\begin{array}{llll}
u_{z}=0, & \sigma_{r z}=0, & m_{\theta z}=0 &
\end{array}
$$

where $C_{0}$ is a constant to be determined by the third equation in Eq. (46). 


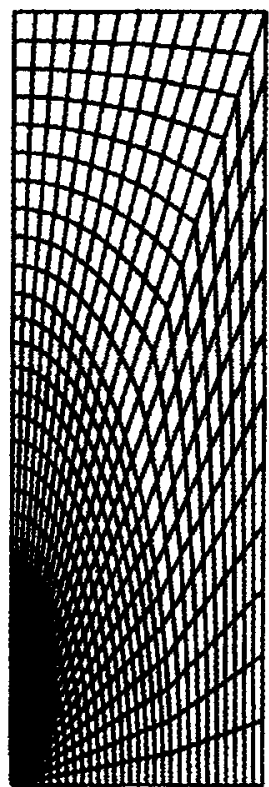

a

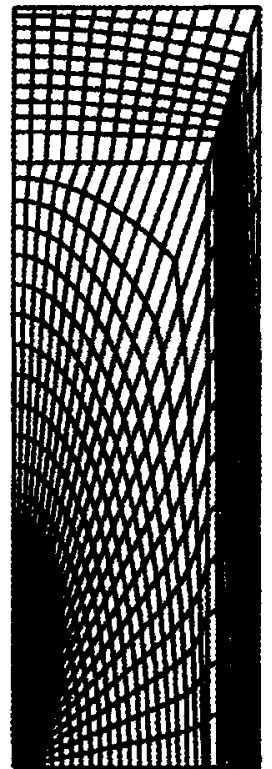

b
Fig. 2. Mesh division of the calculation model: a cylindrical particle reinforced metal-matrix composite; b spheroidal particle reinforced metal-matrix composite

In the present paper, the metal matrix is treated as an elastic-plastic material considering the strain gradient. The particle is treated as an elastic material with Young's modulus $E_{P}$ and Poisson's ratio $\nu_{p}$.

The parameter dependence of the stress-strain relations of the PMMC can be written as

$\frac{\sigma_{C}}{\sigma_{Y}}=F\left(\varepsilon_{C}, \frac{E_{P}}{E}, f_{P}, x, n, \frac{E}{\sigma_{Y}}, \nu, \nu_{P}, \frac{V_{P}^{1 / 3}}{l_{1}}\right)$,

where $E, n, \nu$ are the Young's modulus, the strain hardening exponent and Poisson's ratio of the matrix material, respectively.

In the present paper, we take $\left(E / \sigma_{Y}, \nu, \nu_{P}\right)=(300,0.3,0.3)$ as the same as [20], where $\sigma_{Y}$ is the yield stress of the matrix material. $V_{P}{ }^{1 / 3} / l_{1}$ is a parameter. We know that while the value of $V_{P}{ }^{1 / 3} / l_{1}$ is big and the value of $l_{1}$ keeps constant, it means that the particle size is large; Conversely, when the value is small, the case corresponds to the small particle.

\subsection{Mesh division}

In the present paper, the element form adopted is shown in Fig. 2. The number of nine-node elements is 720 . For comparison, meshes for the two kinds of particles, i.e. spheroidal and cylindrical particles are shown in Fig. 2a and b.

\section{Results for the spheroidal particle case}

In this section, composites reinforced by spheroidal particles are investigated with different particle volume fractions, different particle sizes, different Young's modulus ratio of particle and matrix and different aspect ratios (the ratio of width to height of particle). In all calculations Poisson's ratio is 0.3 for both the particle and the matrix material. 

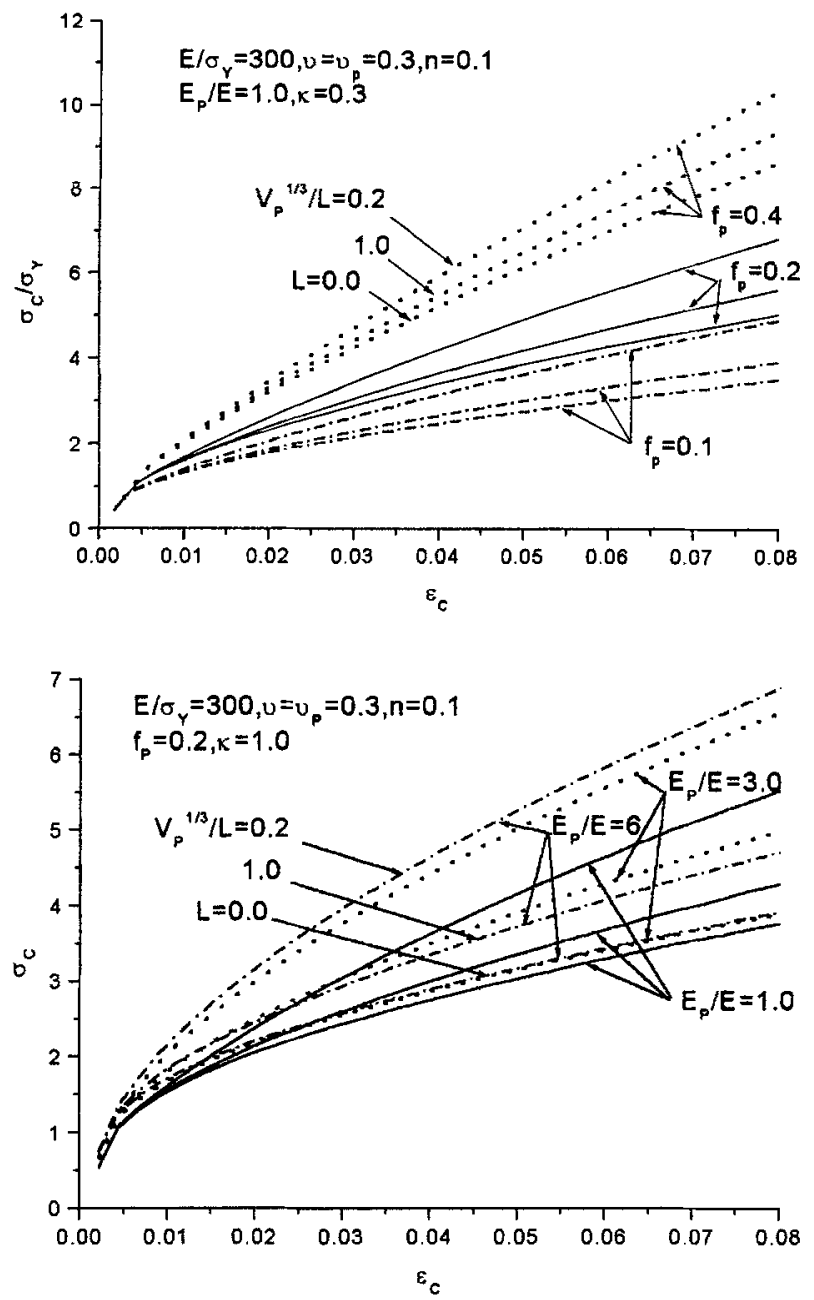

Fig. 3. The dependence of stress strain curves of the composite on the particle volume fractions and the particle sizes, while the other parameters are fixed and Young's modulus ratio of the particle and matrix material is unity.

Fig. 4. The dependence of stress strain curves of the composite on the particle sizes and Young's modulus ratio of the particle and matrix material, while the other parameters are fixed

Figure 3 shows the stress strain curves of the composites for different particle volume fractions and different particle sizes when Young's modulus values of both particle and matrix are equal and the particle aspect ratio is 0.3 . From Fig. 3, one can find that both the particle volume fraction and particle size have strong effects on the composite strength. The larger the particle volume fraction, the larger the PMMC strength, and the smaller the particle size, the larger the PMMC strength.

Figure 4 depicts the dependence of the stress strain relations on the Young's modulus ratios for spherical particles. From Fig. 4, one can find that the stress strain curves are not sensitive to the Young's ratio while the Young's ratio is near to one. But it is very sensitive to the particle sizes, with Young's modulus ratio increasing.

Figure 5 demonstrates that the stress strain curves also depend on the strain hardening exponent of the matrix material. Larger strain hardening exponent, larger the overall composite stress. While the strain hardening exponent increases, the effects of the particle size become stronger.

The results in Fig. 6 include three cases of the aspect ratios: $x=0.1,1.0,10 . x=0.1$ means the particle shape is rather thin; $x=1.0$ corresponds to the spherical shape and $x=10.0$ represents the flat particle shape. When the particle volume fraction is fixed, the composite 

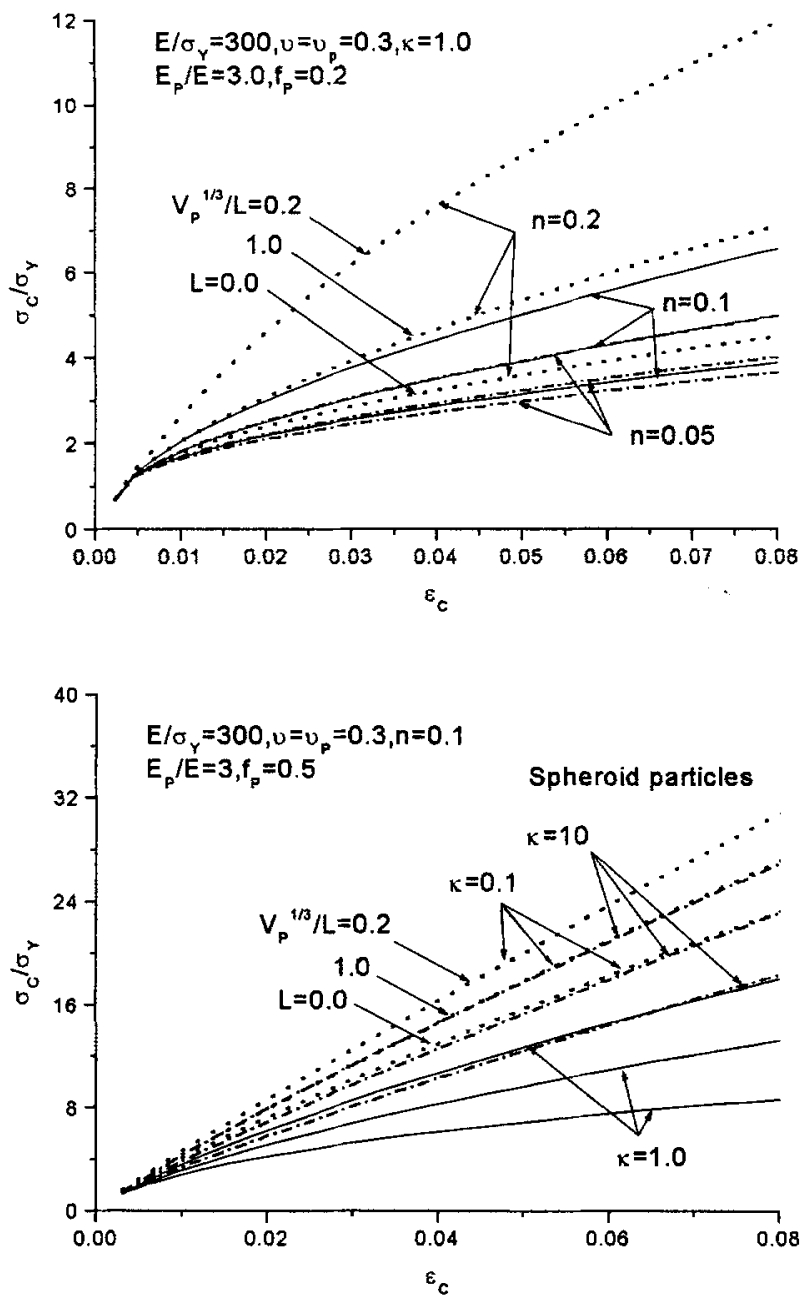

Fig. 5. The dependence of stress strain curves of the composites on the particle sizes and the strain hardening exponents, while the other parameters are fixed

Fig. 6. The dependence of stress strain curves of spheroidal particle reinforced composites on the particle sizes and the particle aspect ratio, while the other parameters are fixed

strengthening is the smallest for the spherical particle case. When the aspect ratio is very large or very small, the composite stresses are almost the same. For the same aspect ratio, the stress is larger while the particle size is smaller, which tends to explain the phenomena found in the experiments [5]-[9].

\section{Results for the cylindrical particle case}

In this section, composites reinforced by cylindrical particles are investigated with different particle volume fractions, different particle sizes, different Young's modulus ratio of particle and matrix and different aspect ratio (the ratio of width to height of particle). In all calculations, the results are similar to those for a spheroidal particle, and the composite strength also depends on the particle size, the particle aspect ratio, Young's modulus ratio of the particle and matrix materials and particle volume fractions, as well as the strain hardening exponent of the matrix material.

Figure 7 shows the comparison of the results of a spheroidal particle with those of a cylindrical particle when all other parameters are fixed. From Fig. 7, one can find that the flow 

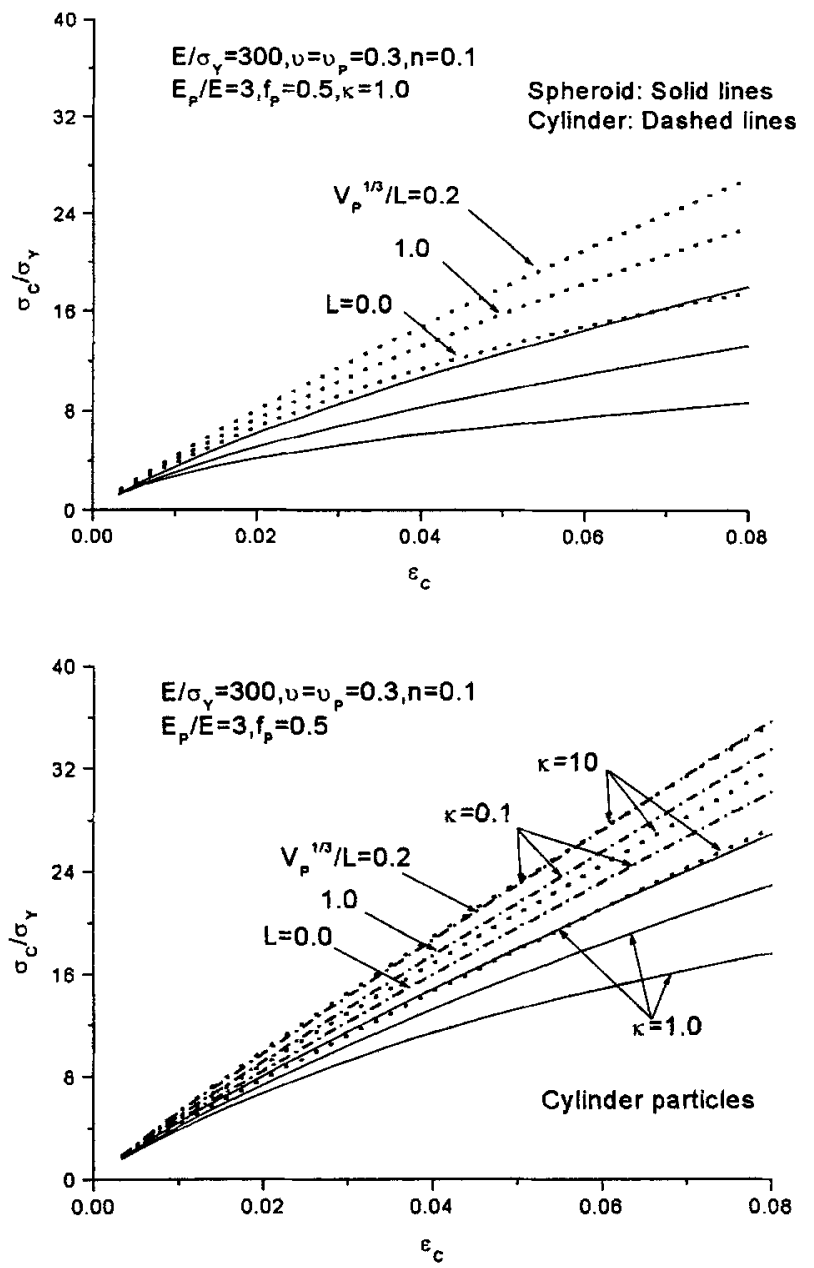

Fig. 7. Comparison of the stress strain curves between spheroidal particle reinforced composites and cylindrical particle reinforced composites, while the other parameters are fixed

Fig. 8. The dependence of stress strain curves of cylindrical particle reinforced composites on the particle sizes and the particle aspect ratio, while the other parameters are fixed

stress for a cylindrical particle reinforced composite is larger than that for a spheroidal particle reinforced composite, which can be found in the conventional composite calculations.

The dependence of stress strain relations on the particle aspect ratio is depicted in Fig. 8. The following conclusions are readily obtained: (i) the composite strengthening is the smallest for $x=1.0$; (ii) When the particle ratio is very low or very high, the difference between two results with size effect considered disappears very quickly.

The influences on the composite strength of the other composite factors in the cylindrical particle reinforced metal-matrix composite show almost the same tendency as those in spheroidal particle reinforced metal-matrix composites. Here the results are omitted.

\section{Comparison with experiments}

In order to determine the internal material scales for a concrete composite, the calculation results will be compared with the experimental results. The above method has been used to analyze a special problem, and the results are compared with the experiment results given by Ling [9]. 


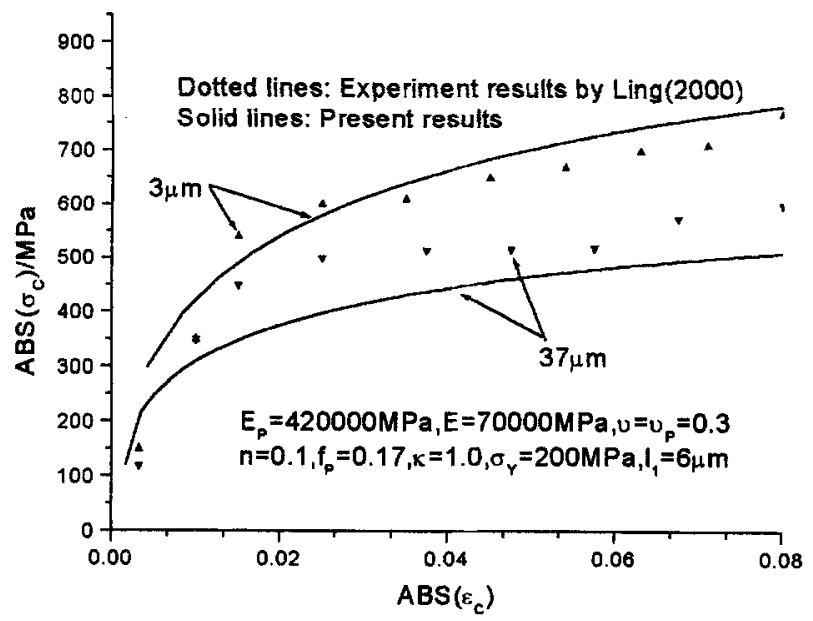

Fig. 9. Comparison between the calculation results and the experimental results given by Ling [9], and the material length scale is predicted, i.e., $l_{1}=6 \mu \mathrm{m}$

A series of uniaxial compression tests of $17 \%$ volume fraction $\mathrm{SiCp} / 2124 \mathrm{Al}$ composites with different particle sizes were carried out by Ling [9]. According to [9], the material parameters are $E=70 \mathrm{GPa}, E_{P}=420 \mathrm{Gpa}, n=0.1, \sigma_{Y}=200 \mathrm{MPa}, x=1.0, f_{P}=0.17$ and $\nu=\nu_{P}=0.3$. The dependence of the compressive stress strain curves on the particle sizes for this lower particle volume fraction from experimental results and calculation results is shown in Fig. 9 for particle radii $3 \mu \mathrm{m}$ and $37 \mu \mathrm{m}$. From Fig. 9, one can find that the present calculation results with length scale $l_{1}=6 \mu \mathrm{m}$ agree with the experimental results by Ling [9]. The calculation has been done on a rectangular unit cell with spherical reinforcement, which is subject to uniformly prescribed boundary conditions; however, in the real experimental sample, the reinforcements have irregular shapes and particle-particle interaction poses a constraint far more complicated than in the simple model we have used. Particles also tend to form clusters, rather than being uniformly distributed as assumed here. These factors may all contribute to the discrepancy remaining between calculation and experimental results.

\section{Conclusions}

The results are different from those in [22], where the local deformation was mainly investigated. The results in the present paper provide some detailed quantitative information on the particle reinforced metal-matrix composites, and the macroscopic stresses are calculated with different composite factors. The results show the dependence of the composite strength on the particle size, Young's modulus of the particle, particle aspect ratio and particle volume fraction, as well as the strain-hardening exponent of the matrix material. In addition, the strength of the PMMC depends on the particle shapes, with the other parameters fixed. The strength of a cylindrical particle reinforced composite is larger than that of a spheroidal particle reinforced composite. Especially, while the particle aspect ratio is very small or very large, the difference between two results is very small, and while the particle aspect ratio is 1 , the strength of PMMC is the smallest. Comparing with the experimental results, the material length scale is predicted, and the calculation results are consistent well with the experimental results. 


\section{Acknowledgement}

This work is supported by the National Natural Science Foundation of China (No. 19704100), National Science Foundation of Chinese Academy of Sciences (Project KJ951-1-20), CAS K. C. Wong Post-doctoral Research Award Fund and the Post-doctoral Science Fund of China.

\section{References}

[1] Christman, T., Needleman, A., Suresh, S.: An experimental and numerical study of deformation in metal ceramic composites. Acta Metall. Mater. 37, 3029-3050 (1989).

[2] Bao, G., Hutchinson, J. W., McMeeking, R. M.: Particle reinforcement of ductile matrices against plastic flow and creep. Acta Metall. Mater. 39, 1871-1882 (1991).

[3] Corbin, S. F., Wilkinson, D. S.: The influence of particle distribution on the mechanical response of a particulate metal-matrix composite. Acta Metall. Mater. 42, 1311-1318 (1994).

[4] Boland, F., Colin, C., Salmon, C. et al.: Tensile flow properties of Al-based matrix composites reinforced with a random planar network of continuous metallic fibers. Acta Mater. 46, 6311-6323 (1998).

[5] Yang, J., Cady, C., Hu, M. S. et al.: Effects of damage on the flow strength and ductility of a ductile Al-alloy reinforced with SiC particulates. Acta Metall. Mater. 38, 2613-2619 (1990).

[6] Kamat, S. V., Rollett, A. D., Hirth, J. P.: Plastic-deformation in Al-alloy matrix-alumina particulate composites. Scripta Metall. Mater. 25, 27-32 (1991).

[7] Lloyd, D. J.: Particle-reinforced aluminum and magnesium matrix composite. Int. Mater. Rev. 39, $1-23$ (1994).

[8] Kiser, M. T., Zok, F. W., Wilkinson, D. S.: Plastic flow and fracture of a particulate metal matrix composite. Acta Mater. 44, 3465-3476 (1996).

[9] Ling, Z.: Deformation behavior and microstructure effect in 2124Al/SuCp composite. J. Comp. Mater. 34, $101-115(2000)$.

[10] Nan, C. W., Clarke, D. R.: The influence of particle size and particle fracture on the elastic/plastic deformation of metal matrix composites. Acta Mater. 44, $3801-3811$ (1996).

[11] Maire, E., Wilkinson, D. S., Embury, D. et al.: Role of damage on the flow and fracture of particulate reinforced alloys and metal matrix composites. Acta Mater. 45, 5261 - 5274 (1997).

[12] Ma, Q., Clarke, D. R.: Size dependent hardness in silver single crystals. J. Mat. Res. 10, 853-863 (1995).

[13] Fleck, N. A., Muller, G. M., Ashby, M. F., Hutchinson, J. W.: Strain gradient plasticity: theory and experiment. Acta Metall. Mater. 42, 475-487 (1994).

[14] Stolken, J. S., Evans, A. G.: A microbend test method for measuring the plasticity length scale. Acta Mater. 46, 5109-5115 (1998).

[15] Elssner, G., Korn, D., Ruehle, M.: The influence of interface impurities on fracture energy of UHV diffusion bonded metal-ceramic bicrystals. Scripta Metall. Mater. 31, 1037-1042 (1994).

[16] Fleck, N. A., Hutchinson, J. W.: A phenomenological theory for strain gradient effects in plasticity. J. Mech. Phys. Solids 41, 1825 - 1857 (1993).

[17] Fleck, N. A., Hutchinson, J. W.: Strain gradient plasticity. Adv. Appl. Mech. 33 (Hutchinson, J. W., Wu, T. Y., eds.), pp. 295-361. New York: Academic Press 1997.

[18] Gao, H., Huang, Y., Nix, W. D., Hutchinson, J. W.: Mechanism-based strain gradient plasticity - I: theory. J. Mech. Phys. Solids 47, 1239 - 1263 (1999).

[19] Wei, Y., Hutchinson, J. W.: Steady-state crack growth and work of fracture for solids characterized by strain gradient plasticity. J. Mech. Phys. Solids 45, 1253-1273 (1997).

[20] Wei, Y.: Particulate size effects in the particle-reinforced metal-matrix composites. Acta Mech. Sin. 17, $45-58$ (2001).

[21] Shu, J. Y., Fleck, N. A.: Strain gradient crystal plasticity: size-dependent deformation of bicrystals. J. Mech. Phys. Solids 47, $292-324$ (1999).

[22] Shu, J. Y., Barlow, L. Y.: Strain gradient effects on microscopic strain field in a metal matrix composite. Int. J. Plast. 16, $563-591$ (2000).

[23] Bassani, J. L., Needleman, A., Giessen, E. Van Der: Plastic flow in a composite: a comparison of nonlocal continuum and discrete dislocation predictions. Int. J. Solids Struct. 38, 833-853 (2001). 
[24] Nix, W. D., Gao, H.: Indentation size effects in crystalline materials: a law for strain gradient plasticity. J. Mech. Phys. Solids 46, 411-425 (1998).

[25] Acharya, A., Shawki, T. G.: Thermodynamic restrictions on constitutive equations for second-deformation-gradient inelastic behavior. J. Mech. Phys. Solids 43, $1751-1772$ (1995).

[26] Aifantis, E. C.: On the microstructural origin of certain inelastic models. Trans. ASME J. Eng. Mater. Technol. 106, 326-330 (1984).

[27] Muhlhaus, H. B., Aifantis, E. C.: The influence of microstructure-induced gradients on the localization of deformation in viscoplastic materials. Acta Mech. 89, 217-231 (1991).

[28] Zbib, H., Aifantis, E. C.: On the localization and postlocalization behavior of plastic deformation. Part I. On the initiation of shear bands. Part II. On the evolution and thickness of shear bands. Part III. On the structure and velocity of Portevin-Le Chatelier bands. Res. Mech. 261-277, 279-292, 293-305 (1989).

[29] Zbib, H., Aifantis, E. C.: On the gradient-dependent theory of plasticity and shear banding. Acta Mech. 92, 209-225 (1992).

[30] Acharya, A., Bassani, J. L.: On non-local flow theories that preserve the classical structure of incremental boundary value problems. In: Micromechanics of plasticity and damage of multiphase materials. IUTAM Symposium, Paris, August 29-September 1, 1995.

[31] Chen, S. H., Wang, T. C.: A new hardening law for strain gradient plasticity. Acta Mat. 48, $3997-4005$ (2000).

[32] Chen, S. H., Wang, T. C.: A new deformation theory for strain gradient effects. Int. J. Plast. (forthcoming 2002).

[33] Chen, S. H., Wang, T. C.: Mode I crack tip with strain gradient effect. Acta Mech. Solida Sin. 13, $290-298(2000)$.

[34] Chen, S. H., Wang, T. C.: Mode I and Mode II crack tip asymptotic fields with strain gradient effects. Acta Mech. Sin. 17, 269-280 (2001).

[35] Chen, S. H., Wang, T. C.: Finite element solutions for plane strain mode I crack with strain gradient effects. Int. J. Solids Struct. (forthcoming 2002).

[36] Chen, S. H., Wang, T. C.: A study of size-dependent microindentation test. (forthcoming).

[37] Smyshlyaev, V. P., Fleck, N. A.: The role of strain gradients in the grain size effect for polycrystals. J. Mech. Phys. Solids 44, 465-495 (1996).

[38] Shu, J. Y., Fleck, N. A.: The prediction of a size effect in microindentation. Int. J. Solids Struct. 35, $1363-1383$ (1998).

[39] Xia, Z. C., Hutchinson, J. W.: Crack tip fields in strain gradient plasticity. J. Mech. Phys. Solids 44, $1621-1648$ (1996).

[40] Shu, J. Y., King, W. E., Fleck, N. A.: Finite elements for materials with strain gradient effects. Int. J. Numer. Methods Eng. 44, 373-391 (1999).

Authors' address: S. H. Chen and T. C. Wang, LNM, Institute of Mechanics, Chinese Academy of Sciences, Beijing, 100080, P.R. China (E-mail: chenshaohua72@hotmail.com) 